\title{
Tratamiento para COVID-19
}

\author{
Treatment for COVID-19 \\ Juana Yagleiry Mercado Rodríguez,* Juliana Taborda Merchán,* \\ Elieth Ochoa García, ${ }^{\ddagger}$ Laura Elena Carreto Binaghi, ${ }^{\S}$ Beatriz Maldonado Tapia,, Emma Rosario García Colín,» \\ Angélica Cecilia Monsiváis Orozco, ${ }^{* *}$ Claudia Garrido Galindo ${ }^{\ddagger}$

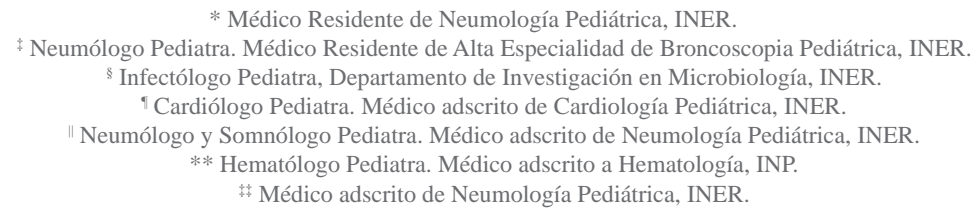

\section{TRATAMIENTO FARMACOLÓGICO}

Actualmente no hay evidencia clínica que permita recomendar un tratamiento específico de la infección causada por SARS-CoV-2 en pacientes con sospecha o confirmación. Sin embargo, con los conocimientos actuales se pueden hacer ciertas recomendaciones para su manejo ${ }^{1}$ como se muestra en la Tabla 1.

\section{TRATAMIENTO SINTOMÁTICO}

Se administra en pacientes confirmados con cuadro clínico leve o en pacientes sospechosos; se recomienda aislamiento social durante 14 días. ${ }^{2}$

Respecto al uso de paracetamol e ibuprofeno, la OMS y algunas autoridades reguladoras, como la AEM, el NHS y la AEMPS, han manifestado que actualmente no existe evidencia que permita afirmar un agravamiento de la infección por COVID-19 con el uso de ibuprofeno u otros fármacos antiinflamatorios no esteroideos (AINEs). Hasta que se generen evidencias adicionales, consideraremos adecuada la aproximación sugerida por el NHS, que, reconociendo la falta de evidencia, no aconseja suspender tratamientos con este medicamento,

Financiamiento: Ninguno.

Conflicto de intereses: Ninguno.

Rev Latin Infect Pediatr 2020; 33 (s1): s42-s51 pero en caso de iniciarlo, prioriza el uso de paracetamol para tratar los síntomas de la infección. ${ }^{3}$

\section{TRATAMIENTO ANTIVIRAL}

\section{Lopinavir/ritonavir}

El lopinavir es un inhibidor de proteasa, utilizado para tratar la infección por VIH con ritonavir como potenciador. Lopinavir/ritonavir tienen actividad frente al coronavirus in vitro, ${ }^{4}$ por lo que se recomienda su uso, con administración temprana, en casos graves que requieran hospitalización. Es importante recordar que los pacientes pueden presentar frecuentemente efectos adversos gastrointestinales al inicio del tratamiento (diarrea y vómito). ${ }^{1}$ Su posología se muestra en la Tabla 2.

\section{Hidroxicloroquina y cloroquina}

Son fármacos inmunomoduladores con estructura química muy similar, que han demostrado tener mecanismos antivirales frente a SARS-CoV-2, entre ellos, bloquean la invasión viral al interferir con la glucosilación de los receptores ECA2, reduciendo la unión entre las células huésped y las proteínas de superficie del coronavirus; también

Citar como: Mercado RJY, Taborda MJ, Ochoa GE, Carreto BLE, Maldonado TB, García CER et al. Tratamiento para COVID-19. Rev Latin Infect Pediatr. 2020; 33 (s1): s42-s51. https://dx.doi.org/10.35366/96670

www.medigraphic.com/infectologiapediatrica 
Rev Latin Infect Pediatr 2020; 33 (s1): s42-s51

Tabla 1: Indicaciones de tratamiento que dependen de la gravedad del cuadro clínico.

\begin{tabular}{|c|c|c|c|}
\hline Cuadro clínico & Radiografía tórax/TCAR & Tratamiento & Actitud \\
\hline $\begin{array}{l}\text { Leve: sin hipoxemia dificultad } \\
\text { respiratoria ausente o leve }\end{array}$ & $\begin{array}{l}\text { No indicada, salvo en grupos } \\
\text { de riesgo }\end{array}$ & Sintomático & $\begin{array}{l}\text { Alta a domicilio salvo grupos } \\
\text { de riesgo }\end{array}$ \\
\hline $\begin{array}{l}\text { Moderado: hipoxemia y/o } \\
\text { dificultad respiratoria moderada }\end{array}$ & $\begin{array}{l}\text { Normal } \\
\text { Cualquier alteración } \\
\text { (opacidades en vidrio despulido } \\
\text { o consolidación) }\end{array}$ & $\begin{array}{l}\text { Sintomático } \\
\text { Hidroxicloroquina o cloroquina + } \\
\text { lopinavir/ritonavir }\end{array}$ & $\begin{array}{l}\text { Ingreso sin tratamiento antiviral } \\
\text { Ingreso con tratamiento antiviral }\end{array}$ \\
\hline $\begin{array}{l}\text { Grave (UTIP): hipoxemia grave, } \\
\text { dificultad respiratoria grave, mal } \\
\text { estado general }\end{array}$ & $\begin{array}{l}\text { Cualquier alteración } \\
\text { (opacidades en vidrio despulido } \\
\text { o consolidación) }\end{array}$ & $\begin{array}{l}\text { Hidroxicloroquina o cloroquina + } \\
\text { lopinavir/ritonavir }\end{array}$ & $\begin{array}{l}\text { Ingreso con tratamiento antiviral } \\
\text { Valorar uso compasivo de } \\
\text { remdesivir o tocilizumab }\end{array}$ \\
\hline
\end{tabular}

inhiben la replicación viral mediante la modificación del $\mathrm{pH}$ celular y la interrupción de la interacción con receptores tipo Toll, entre otros. ${ }^{5}$ La hidroxicloroquina se propuso como mejor tratamiento que la cloroquina, debido a que la hidroxicloroquina atenúa la progresión de COVID-19 mediante la inhibición de la tormenta de citoquinas al reducir la expresión de CD154 en las células T; además tiene menos efectos secundarios. ${ }^{6}$

Estos fármacos tenían las mejores expectativas de eficacia contra COVID-19, ${ }^{7}$ sin embargo, hubo ciertas controversias acerca de su uso, a causa de los efectos secundarios o interacciones farmacológicas, particularmente en pacientes que presentan falla multiorgánica, intervalo QT prolongado en electrocardiograma basal, cardiomiopatía o miopatía documentadas o deficiencia de G6PD. Las dosis recomendadas se muestran en la Tabla 3. En caso de no tener disponible hidroxicloroquina, se había propuesto utilizar cloroquina a dosis que se muestran en la Tabla 4. También se hizo esta recomendación para pacientes menores de $30 \mathrm{~kg}$, considerando que podrían presentar mayores efectos secundarios por la complejidad de la posología.

Actualmente la OMS no recomienda el uso de hidroxicloroquina o cloroquina como tratamiento de la COVID-19.

\section{Remdesivir}

Es un análogo de nucleótido que interfiere con la polimerización del ARN del virus. Inicialmente, se desarrolló para tratar la enfermedad causada por el virus de Ébola, pero se evidenció que tenía actividad in vitro contra varios coronavirus (MERS-CoV y SARS-CoV-2). Se cuentan con datos de seguridad y farmacocinética en un ensayo en fase III de pacientes con Ébola, por lo que actualmente se recomienda su uso en el manejo de SARS-CoV-2,,$^{6,8,9}$ como se muestra en la Tabla 5. Existen criterios específicos para el uso de remdesivir:

a) El paciente debe estar hospitalizado.

b) Debe tener diagnóstico confirmado de SARSCoV-2 por PCR.

c) Debe encontrarse con ventilación mecánica invasiva.

También existen criterios de exclusión para su uso:

a) Fallo multiorgánico.

b) Uso de inotrópicos para mantener presión arterial.

c) Alanina aminotransferasa $(A L T)>5$ veces el límite superior normal.

d) Aclaramiento de creatinina $<30 \mathrm{~mL} / \mathrm{min}$ o diálisis. ${ }^{1}$

Debemos tener en cuenta que es un medicamento de uso compasivo, y no debe utilizarse con otro fármaco experimental (por ejemplo, lopinavir/ ritonavir); si ése es el caso, debe suspenderse mínimo 12 horas antes de la administración de remdesivir. 


\section{TRATAMIENTO ANTIINFLAMATORIO}

\section{Tocilizumab}

Es un agente inmunosupresor del anticuerpo monoclonal inhibidor de la IL-6. ${ }^{2,10}$ Hasta el momento, no hay datos sobre su uso en menores de dos años. Para emplearlo se requiere la determinación de IL-6 predosis y 24 horas después de la última administración se consideran valores elevados $>40 \mathrm{pg} / \mathrm{mL}$. Se recomienda la administración máxima de tres dosis (la segunda 8-12 horas después de la primera y la tercera a las 16-24 horas de la segunda) y evaluar la disminución de la IL-6. ${ }^{1}$ Las dosis recomendadas se muestran en la Tabla 6.

\section{Esteroides sistémicos}

Actualmente, éstos se encuentran contraindicados en las infecciones leves o moderadas de SARSCoV-2, ya que se comenta que podrían aumentar el periodo de replicación viral y no han demostrado mejorar la mortalidad según reportes de SARS y MERS, por lo que el consenso de expertos y la OMS es evitar los esteroides en pacientes con COVID$19,{ }^{9}$ aunque actualmente existe controversia sobre su utilización. ${ }^{11}$ La literatura recomienda un tratamiento sistemático con corticosteroides, particularmente metilprednisolona (1-2 mg/kg/día) durante tres a cinco días, como una terapia adyuvante en pacientes con SIRA grave, sepsis o choque séptico, broncoespasmo, encefalitis o síndrome hemofagocítico. En estos pacientes en particular, la terapia con corticosteroides podría mejorar la saturación de oxígeno $\left(\mathrm{SpO}_{2}\right)$ y la presión arterial de oxígeno $\left(\mathrm{PaO}_{2}\right)$, así como la fracción inspirada de oxígeno $\left(\mathrm{FiO}_{2}\right)^{1,12}$ Estudios en Wuhan compararon en forma retrospectiva la evolución de los pacientes con neumonía grave por COVID-19, que recibieron o no metilprednisolona a dosis de $1-2 \mathrm{mg} / \mathrm{kg} / \mathrm{d}$ durante cinco a siete días; los pacientes que recibieron metilprednisolona tuvieron una mejoría más rápida,

Tabla 2: Dosificación de lopinavir/ritonavir* en pediatría, basada en peso corporal. . $^{1,7}$

\begin{tabular}{|c|c|c|c|}
\hline Peso corporal (kg) & Dosis cada 12 horas (mg/kg) & Días de tratamiento & Efectos adversos \\
\hline $7-15$ & $12 / 3$ & $7-14$ & $\begin{array}{l}\text { Frecuentes: diarrea, náusea, vómito, hipertrigli- } \\
\text { ceridemia e hipercolesterolemia }\end{array}$ \\
\hline $15-40$ & $10 / 2.5$ & $7-14$ & \\
\hline$>40$ & $400 / 100$ & $7-14$ & $\begin{array}{l}\text { Infrecuentes: pancreatitis, prolongación del } \\
\text { segmento QT del electrocardiograma }\end{array}$ \\
\hline \multicolumn{4}{|c|}{ Posología en pacientes de dos semanas a seis meses } \\
\hline & $16 / 4$ & $7-14$ & \\
\hline
\end{tabular}

\begin{tabular}{|c|c|c|c|c|}
\hline Edad & $\begin{array}{l}\text { Dosis cada } 12 \text { horas } \\
\text { (mg/kg/día) }\end{array}$ & Efectos adversos & Dosis máxima* & Duración \\
\hline$<6$ años & 6.5 & $\begin{array}{l}\text { Hipoglucemia, toxicidad } \\
\text { hematológica, musculoesquelética }\end{array}$ & 400 mg/día & 5-14 días \\
\hline$>6$ años & 10 & $\begin{array}{l}\text { y ocular. En combinación con } \\
\text { otros fármacos puede prolongar } \\
\text { el intervalo QT (macrólidos, } \\
\text { ondansetrón) }\end{array}$ & 400 mg/día & $\begin{array}{l}\text { Después del quinto día } \\
\text { disminuya la dosis a la } \\
\text { mitad }\end{array}$ \\
\hline
\end{tabular}




\section{Tabla 4: Pautas de dosificación de cloroquina. ${ }^{6}$}

\begin{tabular}{ccc}
\hline $\begin{array}{c}\text { Dosis cada } 24 \\
\text { horas (mg/kg/día) }\end{array}$ & $\begin{array}{c}\text { Dosis } \\
\text { máxima }\end{array}$ & Duración \\
\hline $\begin{array}{c}\text { Día 1 y 2: } 10 \\
\text { Después: } 5\end{array}$ & 600 mg/día & $\begin{array}{c}5-14 \text { días } \\
\text { Después del segundo } \\
\text { día disminuir la dosis a } \\
\text { la mitad }\end{array}$ \\
\hline
\end{tabular}

Tabla 5: Pautas de dosificación de remdesivir. 5,6,9

\begin{tabular}{lc}
\multicolumn{1}{c}{ Dosis } & Duración \\
\hline Pacientes < $40 \mathrm{~kg}:$ & 10 días \\
Día 1: dosis de carga $5 \mathrm{mg} / \mathrm{kg} \mathrm{IV}$ & \\
Dosis de mantenimiento: $2.5 \mathrm{mg} / \mathrm{kg} \mathrm{IV}$ & \\
cada 24 horas & \\
Pacientes > $40 \mathrm{~kg}:$ & \\
Día 1: dosis de carga $200 \mathrm{mg} \mathrm{IV}$ & \\
Dosis de mantenimiento: $100 \mathrm{mg}$ IV cada & \\
24 horas &
\end{tabular}

IV = intravenoso.

pues el tiempo para que cediera la fiebre fue de 2.06 versus 5.29 días $(p=0.010)$ y el tiempo que requirieron oxígeno suplementario fue de 8.2 días versus 13.5 días $(p<0.001) .{ }^{13}$

\section{USO DE ANTIBIÓTICOS}

No está indicado, a menos que haya evidencia de infección bacteriana. Se ha descrito que el uso conjunto de hidroxicloroquina con azitromicina se asoció con una caída rápida de la carga viral nasofaríngea, disminuyó los días de estancia hospitalaria y favoreció la eliminación más rápida del virus. ${ }^{14}$

Si bien las recomendaciones actuales no apoyan el uso de antibióticos de primera instancia, se debe contemplar su uso en pacientes graves que presenten neumonía de origen no determinado y que esté asociada con cuidados de la salud, sepsis o sospecha de sobreinfección bacteriana, así como esquema de inmunización incompleto. ${ }^{15}$ En un registro de 68 pacientes del norte de China, la tasa de coinfección variaba de 20 a $80 \%$ entre zonas, y los patógenos más frecuentes fueron: virus influenza A y B, Mycoplasma pneumoniae y Legionella pneumophila, por lo que en los pacientes en que se sospeche coinfección, en especial por gérmenes atípicos, se recomienda el uso de macrólidos ${ }^{16}$ como se muestra en la Tabla 7.

\section{Oseltamivir}

Se plantea como opción si existe coinfección con influenza documentada o hasta no tener una prueba de influenza negativa. En vista de la alta incidencia en México de infección por influenza, se recomienda su empleo de forma precoz. ${ }^{17}$ La Tabla 8 muestra su posología.

\section{Interferón alfa-2b (IFN $\alpha$-2b) nebulizado}

Es un potenciador de inmunidad antiviral innata y adaptativa, por lo que se ha utilizado en la infección por SARS-CoV-2. La dosis recomendada es de 100.000-200.000 Ul/kg para casos leves y 200.000$400.000 \mathrm{UI} / \mathrm{kg}$ para casos graves, dos veces al día durante cinco a siete días. ${ }^{2}$ Puede tener efectos secundarios como: anorexia, depresión, insomnio, ansiedad, labilidad emocional, mareo, cefalea,

Tabla 6: Dosis recomendadas de tocilizumab para inhibir el síndrome de liberación de citoquinas.

\begin{tabular}{ll}
\multicolumn{1}{c}{ Peso } & \multicolumn{1}{c}{ Dosis } \\
\hline Pacientes $<30 \mathrm{~kg}$ & $\begin{array}{l}12 \mathrm{mg} / \mathrm{kg} \text { IV (aforar a } 50 \mathrm{~mL} \text { con solución } \\
\text { salina y administrar en } 1 \mathrm{hora})\end{array}$ \\
Pacientes $\geq 30 \mathrm{~kg}$ & $\begin{array}{l}8 \mathrm{mg} / \mathrm{kg} \mathrm{IV} \text { (aforar a } 100 \mathrm{~mL} \text { con solución } \\
\text { salina y administrar en } 1 \mathrm{hora}) \\
800 \mathrm{mg} \text { (en cada infusión) }\end{array}$ \\
Dosis máxima & \\
\hline IV = intravenoso. &
\end{tabular}

Tabla 7: Dosis recomendada de macrólidos. ${ }^{14}$

Azitromicina

\begin{tabular}{lcc}
\hline Dosis cada 24 horas (mg/kg/día) & Duración & Dosis máxima \\
\hline $\begin{array}{l}\text { Día 1: } 10 \\
\text { Día 2-5: } 5\end{array}$ & 5 días & $\begin{array}{c}500 \text { mg cada 24 } \\
\text { horas }\end{array}$ \\
\hline \multicolumn{3}{c}{ Claritromicina } \\
\hline Dosis cada 12 horas (mg/kg/día) & \\
\hline 15 & 5 días & $\begin{array}{c}1 \text { gramo cada 24 } \\
\text { horas }\end{array}$
\end{tabular}




\begin{tabular}{|ccc|}
\hline \multicolumn{3}{|c}{ Tabla 8: Dosis recomendada de oseltamivir. ${ }^{17}$} \\
\hline Peso $(\mathrm{kg})$ & $\begin{array}{c}\text { Dosis cada } 12 \\
\text { horas }(\mathrm{mg})\end{array}$ & Duración \\
\hline$<15$ & 30 & 5 días \\
$15-23$ & 45 & \\
$24-40$ & 60 & \\
40 & 75 & \\
\hline
\end{tabular}

náusea, vómito, diarrea, dolor abdominal, alopecia, dermatosis, mialgias, artralgias, dolor musculoesquelético, inflamación y reacción en punto de inyección, fatiga, rigidez, pirexia, síntomas pseudogripales, malestar general e irritabilidad.

En nuestro medio no está comercializado, aunque algunas guías de China lo recomiendan para casos leves y graves; sin embargo, no podemos hacer una fuerte recomendación de su uso, debido a la falta de ensayos clínicos.

\section{PLASMA CONVALECIENTE}

La Administración de Drogas y Alimentos de los Estados Unidos ha aprobado el uso de plasma de pacientes recuperados para tratar a los enfermos graves por COVID-19.

El plasma de personas que se han recuperado contiene anticuerpos (IgG e IgM) contra SARSCoV-2 y podría ser efectivo contra la infección. Ha sido estudiado en brotes de otras infecciones de vía respiratoria como la gripe causada por influenza AH1N1, SARS y MERS. ${ }^{18}$

Los donantes deben cumplir las siguientes características: temperatura corporal normal durante más de tres días, resolución de los síntomas del tracto respiratorio, dos resultados consecutivos negativos para SARS-CoV-2 por ensayo de RT-PCR con intervalo de un día de toma de la muestra, título específico de anticuerpos ELISA superior a 1:1,000 y títulos de anticuerpos neutralizantes mayor de $40 . .^{19}$

Henguang y colaboradores,,$^{20}$ en una serie de casos de cinco pacientes graves con COVID-19 y que fueron tratados con plasma convaleciente, informaron una mejoría clínica caracterizada por normalización de la temperatura corporal al tercer día en cuatro de cinco pacientes, disminución de la puntuación SOFA e incremento en el cociente $\mathrm{PaO}_{2} / \mathrm{FiO}_{2}$ en 12 días; la carga viral también disminuyó y negativizaron.
A los 12 días posteriores de la transfusión, hubo incremento en los títulos de anticuerpos neutralizantes (previo 40-60 y 80-320 después) así como del ELISA específico para SARS-CoV-2 y resolución del SDRA en cuatro pacientes, de los cuales se pudo retirar el soporte ventilatorio en tres de ellos.

Debemos considerar algunas limitaciones para su uso: el número de pacientes tratados es reducido y han recibido tratamiento estándar, lo que pudiera explicar la evolución favorable. Hacen falta ensayos clínicos para poder recomendar su empleo, pues hasta el momento no hay experiencia en pacientes pediátricos.

\section{DESARROLLO DE VACUNAS}

Aún no se ha desarrollado alguna inmunización específica y las vacunas se encuentran en fase de investigación; la mayoría se basan en la proteína S que se une al receptor celular y media la actividad de fusión con la membrana celular.

Experimentos previos en modelos animales y ensayos de fase I realizados con vacunas frente a los virus SARS y MERS han mostrado que las vacunas basadas en la proteína $S$ inducen anticuerpos que previenen una infección posterior.

\section{TERAPIA ANTICOAGULANTE}

Se debe administrar anticoagulación profiláctica y realizar una vigilancia estrecha para detectar y tratar los eventos tromboembólicos, y también se debe continuar el tratamiento anticoagulante durante todo el proceso terapéutico. Los pacientes con una puntuación de coagulopatía inducida por sepsis (SIC, por sus siglas en inglés) $\geq 4$ puntos o un DD mayor de $3,000 \mathrm{ng} / \mathrm{mL}$ han mostrado una reducción en la mortalidad cuando recibieron profilaxis con heparinas fraccionadas o no fraccionadas. ${ }^{21,22}$

En niños mayores de 12 años, se pueden seguir las guías de anticoagulación propuestas para pacientes adultos, las cuales están ajustadas a dosis ponderal, principalmente para aquellos pacientes con peso menor a $40 \mathrm{~kg}$ con una dosis profiláctica de enoxaparina de $1 \mathrm{mg} / \mathrm{kg} /$ día cada 24 horas hasta 1.5 $\mathrm{mg} / \mathrm{kg} /$ día dividido en dos dosis. ${ }^{17}$ En niños menores de 12 años se deben establecer los factores de riesgo y valorar la anticoagulación profiláctica en aquellos niños con riesgo alto de trombosis, es decir, aquéllos con tres o más factores de riesgo y enfermedad grave por COVID 19, con excepción de aquéllos 
Rev Latin Infect Pediatr 2020; 33 (s1): s42-s51

con un riesgo elevado de sangrado. En pacientes con trombosis documentada, se debe administrar anticoagulación terapéutica con base en las guías de anticoagulación.

Se debe valorar si se agrega aspirina en el caso de que ocurra trombosis, a pesar de las dosis terapéuticas de enoxaparina. Se consideran pacientes con riesgo alto de trombosis aquéllos con COVID-19 grave, pacientes con DD > 3,000 $\mathrm{ng} / \mathrm{m}$, score para CID $\geq 5$ o de $\mathrm{SIC} \geq 4$, trombofilia conocida, antecedente de trombosis y cáncer activo en ausencia de riesgo de hemorragia, así como pacientes que tienen enfermedad grave por COVID-19 (PCR > $150 \mathrm{mg} / \mathrm{L}, \mathrm{DD}>1,500 \mathrm{ng} / \mathrm{mL}$, IL-6 $>40 \mathrm{pg} / \mathrm{mL}$, ferritina $>1,000 \mathrm{ng} / \mathrm{mL}$, linfopenia $<800 / \mathrm{mm}^{3}$ ), elevación de proteínas procoagulantes incluyendo niveles altos de fibrinógeno, factor VIII y factor von Willebrand. Al momento del egreso, se recomienda continuar con anticoagulación profiláctica con enoxaparina $40 \mathrm{mg}$ al día o apixaban 2.5 mg cada 12 horas por siete a 14 días y fomentar la deambulación. ${ }^{21,23}$

\section{INTERACCIONES FARMACOLÓGICAS}

La cloroquina y la hidroxicloroquina son antimaláricos usados en el manejo contra SARS-CoV-2, a causa de su efecto antiviral e inmunomodulador, aunque la hidroxicloroquina tiene un mejor perfil de seguridad respecto a la cloroquina. ${ }^{1}$ Podemos encontrar dentro de los efectos adversos a considerar de ambos medicamentos: anemia, trombocitopenia, leucopenia, prolongación del intervalo QT o bradicardia. Estos dos últimos se han visto potenciados con la administración conjunta de otros medicamentos como los macrólidos y quinolonas. ${ }^{2}$ Estos datos son de relevancia clínica para el manejo del SARS-CoV-2, ya que se ha evidenciado una reducción de la carga viral con el uso de hidroxicloroquina más azitromicina, ${ }^{3}$ por lo que se recomienda la toma diaria de electrocardiograma y evaluación hemodinámica en este grupo de pacientes. ${ }^{4}$

El tocilizumab es un anticuerpo monoclonal, inhibidor de la interleucina 6 (IL-6), un mediador proinflamatorio liberado del síndrome de liberación de citoquinas, presente en la infección por SARSCoV-2, ${ }^{6,7}$ que al combinarse junto con cloroquina o hidroxicloroquina podría prolongar el intervalo QT, sin embargo, aún faltan más estudios al respecto. ${ }^{8}$

En cuanto al uso de lopinavir/ritonavir, éstos han demostrado tener actividad antiviral contra el
SARS-CoV-2. La administración del ritonavir tiene como objetivo la inhibición del citocromo P450, lo que favorece el aumento en las concentraciones y vida media del lopinavir y así se puede favorecer el efecto antiviral. Entre las complicaciones más frecuentes, se mencionan las gastrointestinales, ${ }^{5,10}$ así como la prolongación del intervalo QT e interacciones farmacológicas a medicamentos que usen como sustrato el CYP3A, ya que inhiben al mismo; ${ }^{8}$ entre estos medicamentos se encuentra el remdesivir, un análogo de nucleótido que, al parecer, interfiere con la polimerización del ARN del virus y tiene una aparente disminución de la carga viral del SARSCoV- $2,{ }^{9}$ el cual aumenta su concentración en plasma y aumenta el riesgo de presentar efectos adversos del medicamento al inhibirse la CYP3A por parte del lopinavir/ritonavir, ${ }^{8}$ lo que puede ocasionar alteraciones en la función renal y hepática; sin embargo, la seguridad y el perfil de efectos secundarios de remdesivir en pacientes con COVID-19 requieren una evaluación adecuada de ensayos controlados con placebo. ${ }^{9,11}$

Existe evidencia de un mayor compromiso por SARS-CoV-2 en pacientes con diagnóstico de hipertensión arterial sistémica, ${ }^{11}$ por lo que el uso y continuidad del tratamiento con inhibidores de la enzima convertidora de angiotensina (IECAS) ha sido controvertido, ya que se ha demostrado que puede aumentar la expresión de los receptores ACE2 en diversos tejidos como pulmón, corazón, riñón y a nivel gastrointestinal, existiendo un riesgo potencialmente mayor de desarrollar infección por SARS-CoV-2 y complicaciones a nivel cardiovascular; sin embargo, no hay datos experimentales o clínicos que apoyen esta hipótesis, por lo que aún no se ha demostrado que tenga beneficio su suspensión. ${ }^{4}$

Ningún estudio realizado ha confirmado la relación entre los antiinflamatorios no esteroideos (AINEs), ${ }^{12}$ como el ibuprofeno y la exacerbación de la infección por COVID 19, por lo que no se ha indicado la suspensión de los mismos en pacientes con enfermedades crónicas o agudas que incluyen estos medicamentos como parte del tratamiento; ${ }^{13}$ no obstante, se presume que pudieran enmascarar algunos síntomas de infecciones graves y, así, retrasar el diagnóstico e inicio del tratamiento, como es el caso de la infección por SARS-CoV-2. Se prefiere, de entrada, el manejo sintomático de la fiebre con paracetamol, ${ }^{14}$ hasta contar con mayor evidencia. 


\section{OXIGENOTERAPIA E INHALOTERAPIA}

Tanto la oxigenoterapia como la inhaloterapia son estrategias fundamentales en el soporte vital de pacientes con COVID-19 en estado crítico, los cuales manifiestan un déficit agudo de oxígeno con cambios a nivel sistémico, principalmente respiratorio, cerebral y cardiovascular. Los dispositivos para la administración de oxígeno y de fármacos inhalados o nebulizados dependen de la condición clínica del paciente, que en estos casos tienen particularidades muy importantes.

La situación actual de esta pandemia está generando dificultades sin precedentes en todos los sistemas de salud a nivel mundial; a pesar de que sólo un porcentaje pequeño puede requerir un manejo avanzado de la vía aérea, la gran cantidad de pacientes infectados puede ocasionar escases de los recursos básicos de oxigenoterapia e inhaloterapia, por lo cual debemos optimizarlos y conocer sus indicaciones.

La oxigenoterapia está recomendada por la OMS y el CDC como terapia de primera línea en pacientes con COVID-19. ${ }^{24}$ El manejo clínico de estos pacientes está basado principalmente en las medidas de soporte para la insuficiencia respiratoria al incrementar la concentración de oxígeno inspirado que garantice un intercambio de gases adecuado, controle la hipoxemia y evite sus consecuencias. ${ }^{25}$

Previamente al inicio del aporte de oxígeno suplementario, debemos realizar una evaluación completa y diferenciar el tipo de hipoxia, los antecedentes patológicos y los datos clínicos según la gravedad, ya que ello nos permite hacer una selección correcta del sistema de administración de oxígeno, el cual se clasifica según la cantidad de la mezcla gaseosa que llega al paciente, es decir, si es de alto flujo o de bajo flujo. ${ }^{26}$ El objetivo de la administración de oxígeno suplementario será mantener la saturación de oxígeno $\left(\mathrm{SpO}_{2}\right)$ mayor a $90 \%$ al nivel de la Ciudad de México y a $93 \%$ a nivel del mar. $^{24}$ Las fuentes de oxígeno son diversas y permiten cubrir todas las necesidades en diferentes ambientes, ya sea un paciente hospitalizado o atención domiciliaria; sin embargo, los dispositivos de administración e interfaces tienen indicaciones precisas en pacientes con COVID-19 y nos centraremos en la oxigenoterapia en ambiente hospitalario. Debemos tener presente que todos los dispositivos son potenciales generadores de aerosoles y que se debe usar equipo de protección personal (EPP).

\section{Dispositivos de administración de oxígeno}

A. Bajo flujo: dependen de varios factores, como el flujo del oxígeno, el patrón respiratorio, el volumen corriente y las características anatómicas del paciente; es una mezcla de oxígeno variable, por lo que la $\mathrm{FiO}_{2}$ es impredecible y en ocasiones insuficiente. ${ }^{26}$

1. Cánulas nasales. Con este dispositivo se incrementa la $\mathrm{FiO}_{2}$ de 3 a $4 \%,{ }^{27}$ pero puede variar según el volumen corriente. Puede ser útil en pacientes con hipoxemia leve, aunque su principal desventaja es que induce una dispersión importante del aire exhalado aun con bajo flujo, esto es, hasta $30 \mathrm{~cm}$ con flujo de $1 \mathrm{~L} / \mathrm{min} .{ }^{24}$

2. Mascarilla facial simple. Requiere como mínimo 5-10 L/min para evitar reinhalación y con ella se puede suministrar una $\mathrm{FiO}_{2}$ de $35-60 \%$. $^{25}$ Una característica importante es que cuenta con ventanas laterales que permiten la salida del aire exhalado, lo que genera aerosoles a una distancia máxima de $40 \mathrm{~cm}$ a $10 \mathrm{~L} / \mathrm{min}^{24}$

3. Mascarilla con reservorio. Estas mascarillas son una forma segura de oxigenoterapia, ya que proporcionan oxígeno en altas concentraciones, limitan la dispersión de las gotas y previenen la hipercapnia siempre que el reservorio esté inflado, para lo cual necesita un flujo de 6 a $10 \mathrm{~L} / \mathrm{min}$. La distancia máxima de dispersión de aerosoles es de menos de 10 $\mathrm{cm}$ con flujo a $10 \mathrm{~L} / \mathrm{min}$, por lo que la recomendamos. ${ }^{24,25}$

B. Alto flujo: se caracterizan por utilizar el efecto Venturi, en el cual se hace pasar un flujo de oxígeno a gran velocidad por un orificio central, arrastrando gas ambiental por otro orificio por succión de los alrededores de la mascarilla y, por tanto, la $\mathrm{FiO}_{2}$ es controlada y conocida, además de independiente del esfuerzo respiratorio del paciente. ${ }^{26}$

1. Mascarilla Venturi. Es el sistema más representativo de los dispositivos de alto flujo; suministra una $\mathrm{FiO}_{2}$ exacta desde 24 a $60 \%$ con flujos de 2 hasta $15 \mathrm{~L} / \mathrm{min} .^{28}$ Según estudios realizados en un maniquí humano, la distancia máxima de dispersión es de $40 \mathrm{~cm}$ con $\mathrm{FiO}_{2}$ de $24 \% .^{24}$

2. Puntas de alto flujo. Este sistema requiere flujos altos entre $6-10 \mathrm{~L} / \mathrm{min}$ y puede utilizarse como un paso previo al uso de la ventilación no invasiva. ${ }^{29}$ Su principal característica es que 
permite un lavado del espacio muerto anatómico y disminuye las resistencias inspiratorias en la vía aérea superior. ${ }^{25}$ Existe controversia con respecto a su uso en pacientes COVID19, debido a la generación de aerosoles, sin embargo, estudios recientes han demostrado que con una adecuada interfaz y con flujo a 60 L/min (en maniquís) la dispersión del aire exhalado es de $17 \mathrm{~cm}$; además se sugiere colocar mascarillas quirúrgicas encima de las puntas de alto flujo. 24,30

3. Tienda facial. Si se le acopla un sistema Venturi, funciona como dispositivo de alto flujo y puede utilizarse en pacientes que no toleran las mascarillas faciales o puntas nasales. ${ }^{28}$

4. Campana de oxígeno. Es un dispositivo cerrado y compacto, al cual se le acopla un sistema Venturi; suele ser más utilizado en lactantes. ${ }^{28}$

5. Mascarilla de traqueostomía. Amerita flujos altos, proporciona un alto grado de humedad y debe eliminarse de ella la condensación cada dos horas; además, el orificio central de la mascarilla permite la aspiración de secreciones. ${ }^{28}$

6. Ventilación mecánica no invasiva. En relación con esta terapia de ventilación, podría ser útil en algunos casos, siempre y cuando estén aseguradas las medidas preventivas, puesto que se debe limitar la transmisión de la infección tanto al personal de salud como a otros pacientes. En capítulos posteriores profundizaremos con detalle sobre aspectos importantes de esta estrategia de ventilación.

La administración de medicamentos por la vía inhalada proporciona una acción rápida y directa, reduciendo las reacciones adversas y es clave en

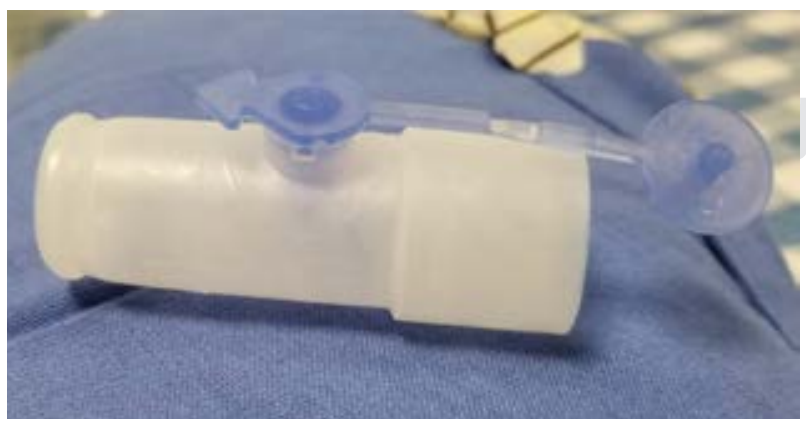

Figura 1: Adaptador para aplicar los medicamentos en dosis medida para pacientes bajo ventilación mecánica.

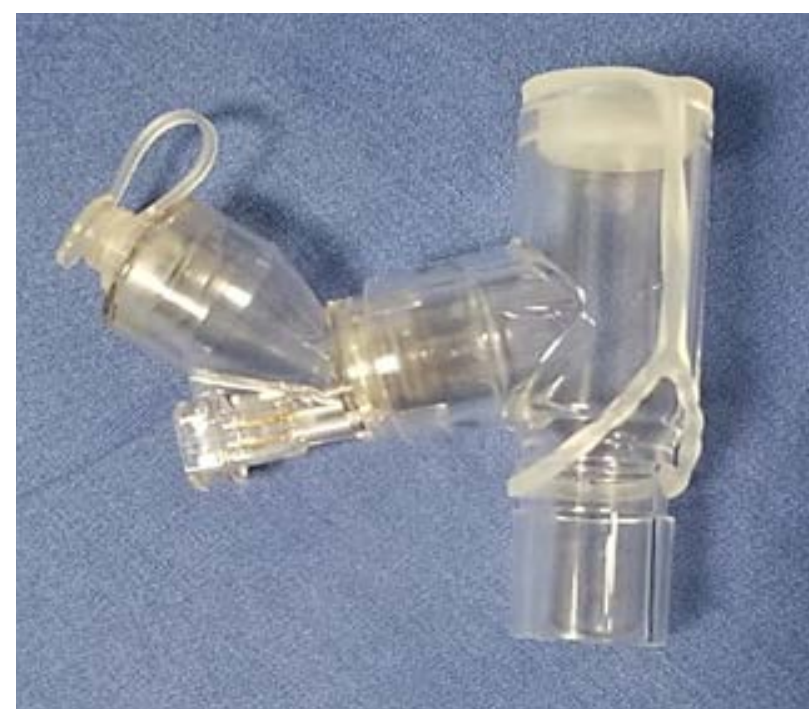

Figura 2: Nebulizador de tipo malla vibratoria.

el manejo de las enfermedades pulmonares; sin embargo, la estrategia de medicamentos nebulizados debe evitarse en la medida de lo posible en el cuidado de pacientes COVID-19, ya que el perfil de seguridad es muy escaso y existe un alto riesgo de contagio, debido al potencial de generar un alto volumen de aerosoles que pueden ser propulsados a una distancia mayor que la involucrada en el patrón de dispersión natural. En caso de pacientes con asma o EPOC, se recomienda el uso de dispositivos de dosis medida con aerocámara o polvo seco cuando se encuentran estables, y en pacientes bajo ventilación mecánica que requieran broncodilatador o esteroides, se recomienda aplicarse con un adaptador que va en la rama inspiratoria del circuito de ventilación (Figuras 1 y 2). En caso de que el medicamento no se encuentre en MDI o no se cuente con dicho adaptador, se sugiere utilizar un nebulizador de tipo malla vibratoria, ya que este dispositivo es el que tiene menor riesgo de generar aerosoles e incrementar el depósito pulmonar; además reduce la liberación del fármaco al aire ambiente y, por lo tanto, hay un menor riesgo de contagio al personal de salud. . $4,29,31^{2}$

Se desaconsejan los sistemas jet, a causa de la mayor capacidad de dispersión de partículas al ambiente. ${ }^{32}$

Consideramos que el dispositivo ideal será la mascarilla con reservorio y, de ser posible, se debe usar, sobre estos dispositivos, una mascarilla quirúrgica que evite la dispersión del virus. La ventilación mecánica no invasiva se deberá considerar como 
última opción antes de la ventilación mecánica invasiva, siempre y cuando se cumplan con las medidas de seguridad para el personal de salud. Recomendamos el uso de dispositivos de dosis medida y polvo seco para pacientes estables o aplicar el dispositivo de dosis medida (MDI) con un adaptador; como última alternativa, recomendamos usar nebulizador de tipo malla vibratoria en pacientes bajo ventilación mecánica cuando sea indispensable la aplicación de medicamentos mediante nebulizador.

La terapia respiratoria es vital en el manejo de enfermedades pulmonares, sin embargo, ante la situación mundial se requiere una valoración y análisis exhaustivo de los riesgos y beneficios para la decisión del dispositivo de soporte respiratorio, considerando los riesgos que cada uno genera para la infección del personal de salud.

\section{CONCLUSIONES}

Las recomendaciones sobre los tratamientos que emitimos están basadas en revisiones bibliográficas y, principalmente, en estudios efectuados con población adulta. Actualmente se encuentran en fase de investigación un gran número de medicamentos y terapias, por lo que este documento está sujeto a cambios conforme avancen dichas investigaciones.

Consideramos que el dispositivo ideal será la mascarilla con reservorio y, de ser posible, se debe usar sobre estos dispositivos una mascarilla quirúrgica que evite la dispersión del virus. La ventilación mecánica no invasiva se deberá considerar como última opción antes de la ventilación mecánica invasiva, siempre y cuando se cumplan las medidas de seguridad para el personal de salud. Recomendamos el uso de dispositivos de dosis medida y polvo seco para pacientes estables o aplicar dicho dispositivo con un adaptador; como última alternativa, se debe usar un nebulizador de tipo malla vibratoria en pacientes que estén bajo ventilación mecánica, cuando sea indispensable la aplicación de medicamentos mediante nebulizador.

La terapia respiratoria es vital en el manejo de enfermedades pulmonares, sin embargo, ante la situación mundial se requiere una valoración y análisis exhaustivo de los riesgos y beneficios para la decisión del dispositivo de soporte respiratorio, considerando los peligros que cada uno genera para la infección del personal de salud.

\section{REFERENCIAS}

1. Calvo Rey C, García-López Hortelano M, Tomás Ramos J, Baquero Navarro F, Navaro M et al. Documento de manejo clínico del paciente pediátrico con infección por SARS-CoV-2. Extracto del Documento de Manejo Clínico del Ministerio de Sanidad [Internet]. Sociedad Española de Infectología Pediatrica; 2020 [actualizado 18 de agosto 2020]. p. 1-17. Disponible en: https://www.analesdepediatria.org/contenidos/ pdf/Recomendaciones_pediatricas_Covid1.pdf

2. Acuña Cordero R, Bejarano Melo MA, Niederbacher Velásquez J, Pulido Fentanes S, Restrepo Gualteros SM, Villamil Osorio M. COVID-19 en niños, Colombia, versión 2 [Internet]. Asociación Colombiana de Neumología Pediátrica. 2020 [actualizado 1 de abril 2020]. pp. 1-18. Disponible en: https://scp.com.co/wp-content/ uploads/2020/04/Coronavirus-Version-2.pdf.pdf

3. PAHO. Ibuprofeno y COVID-19. 18 de marzo de 2020 [Internet]. Disponible en: https://www.paho.org/es/ documentos/ibuprofeno-covid-19-washington-dc-18marzo-2020

4. Sheahan TP, Sims AC, Leist SR, Schäfer A, Won J, Brown AJ et al. Comparative therapeutic efficacy of remdesivir and combination lopinavir, ritonavir, and interferon beta against MERS-CoV. Nat Commun [Internet]. 2020; 11 (1): 222. Available from: https://www.nature.com/articles/s41467019-13940-6.pdf

5. Ko WC, Rolain JM, Lee NY, Chen PL, Huang CT, Lee PI et al. Arguments in favor of remdesivir for treating SARSCoV-2 infections. Int J Antimicrob Agents [Internet]. 2020; 55 (4): 105933. Available from: https://www.ncbi.nlm.nih. gov/pmc/articles/PMC7135364/pdf/main.pdf

6. Fung KL, Chan PL. Comment on: COVID-19: a recommendation to examine the effect of hydroxychloroquine in preventing infection and progression. J Antimicrob Chemother [Internet]. 2020; 75 (7): 2016-2017. Available from: https://watermark.silverchair.com/dkaa114.pdf?token=AQE CAHi208BE49Ooan9kkhW_Ercy7Dm3ZL_9Cf3qfKAc485ysgAAAoUwggKBBgkqhkiG9w0BBwagggJyMIICbgIBADCC AmcGCSqGSIb3DQEHATAeBglghkgBZQMEAS4wEQQMc4x2Yqwh72fM9crwAgEQgllCOIO48ZhtTNBuT4CUDqQKdX5BJeQzz2ylqhCQHQxXFI5WBU3

7. Ministerio de Sanidad de España. Tratamientos disponibles para el manejo de la infección respiratoria por SARS-CoV-2 [Internet]. Agencia Española de Medicamentos y productos. 2020 [actualizado 9 julio 2020]. Disponible en: https://www. aemps.gob.es/la-aemps/ultima-informacion-de-la-aempsacerca-del-covid-19/tratamientos-disponibles-para-elmanejo-de-la-infeccion-respiratoria-por-sars-cov-2/

8. Mulangu S, Dodd LE, Davey RT, Mbaya OT, Proschan M, Mukadi $\mathrm{D}$ et al. A randomized, controlled trial of Ebola virus disease therapeutics. N Engl J Med. 2019; 381 (24): 2293-2303.

9. Moore JB, June $\mathrm{CH}$. Cytokine release syndrome in severe COVID-19. Science [Internet]. 2020; 368 (6490): 473474. Disponible en: https://science.sciencemag.org/ content/368/6490/473/tab-pdf

10. Xu X, Han M, Li T, Sun W, Wang D, Fu B et al. Effective treatment of severe COVID-19 patients with tocilizumab. Proc Natl Acad Sci U S A [Internet]. 2020; 117 (20): 1097010975. Disponible en: https://www.pnas.org/content/ pnas/117/20/10970.full.pdf

11. McGonagle D, Sharif K, O'Regan A, Bridgewood C. The role of cytokines including interleukin-6 in COVID-19 induced 
Rev Latin Infect Pediatr 2020; 33 (s1): s42-s51

pneumonia and macrophage activation syndrome-like disease. Autoimmun Rev [Internet]. 2020; 19 (6): 102537. Available from: https://reader.elsevier.com/reader/sd/pii/S1 568997220300926?token=F43051E26D5C53F4DE8F2578 92A8DBF643EB879C8411430E98984B128701748885331 57A0154AFCEE2A391CA5041D254

12. Zhou W, Liu Y, Tian D, Wang C, Wang S, Cheng J et al. Potential benefits of precise corticosteroids therapy for severe 2019-nCoV pneumonia. Signal Transduct Target Ther. 2020; 5 (1): 17-19.

13. Wang $Y$, Jiang $W, H e ~ Q$, Wang $C$, Wang $B$, Zhou $P$ et al. Early, low-dose and short-term application of corticosteroid treatment in patients with severe COVID-19 pneumonia: single-center experience from Wuhan, China. medRxiv [Internet]. 2020. doi: https://doi.org/10.1101/2020.03.06.200 32342. Disponible en: https://www.medrxiv.org/content/10.1 101/2020.03.06.20032342v1.full.pdf+html

14. Gautret P, Lagier JC, Parola P, Thuan Hoang V, Meddeb Line et al. Clinical and microbiological effect of a combination of hydroxychloroquine and azithromycin in 80 COVID-19 patients with at least a six-day follow up: a pilot observational study. Travel Med Infect Dis [Internet]. 2020; 34: 101663. Disponible en: https://www.ncbi.nlm.nih.gov/ pmc/articles/PMC7151271/pdf/main.pdf

15. González-Castro A, Escudero-Acha P, Peñasco Y, Leizaola O, Martínez de Pinillos Sánchez V. Cuidados intensivos durante la epidemia de coronavirus 2019. Med Intensiva [Internet]. 2020; 44 (6): 351-362. Disponible en: https://www. medintensiva.org/contenidos/pdf/MEDIN 1476.pdf

16. Xing Q, Li G, Xing Y, Chen T, Li W, Ni W et al. Precautions are needed for COVID-19 patients with coinfection of common respiratory pathogens. medRxiv [Internet]. 2020. Disponible en: https://www.medrxiv.org/content/10.1101/20 20.02.29.20027698v2.full.pdf

17. Calvo C, García López-Hortelano M, de Carlos Vicente JC, Vázquez Martínez JL. Recomendaciones sobre el manejo clínico de la infección por el «nuevo coronavirus» SARS-CoV2. Grupo de trabajo de la Asociación Española de Pediatría (AEP). An Pediatr (Barc) [Internet]. 2020; 92 (4): 241.e1-241.e11. Disponible en: https://www. analesdepediatria.org/es-pdf-S169540332030076X

18. Tanne JH. Covid-19: FDA approves use of convalescent plasma to treat critically ill patients. BMJ [Internet]. 2020. doi: 10.1136/bmj.m1256. Disponible en: https://www.bmj. com/content/bmj/368/bmj.m1256.full.pdf

19. Duan K, Liu B, Li C, Zhang H, Yu T, Qu J, Zhou M et al. Effectiveness of convalescent plasma therapy in severe COVID-19 patients. Proc Natl Acad Sci U S A [Internet]. 2020; 117 (17): 9490-9496. Disponible en: https://www.pnas.org/ content/pnas/early/2020/04/02/2004168117.full.pdf

20. Shen C, Wang Z, Zhao F, Yang Y, Li J, Yuan J et al. Treatment of 5 critically ill patients with COVID-19 with convalescent plasma. JAMA [Internet]. 2020; 323 (16): 1582-1589. Disponible en: https://jamanetwork.com/ journals/jama/fullarticle/2763983

21. Connors J, States U, Levy J, States U. COVID-19 and its implications for thrombosis and anticoagulation COVID-19 and its implications for thrombosis and anticoagulation. Blood [Internet]. 2020; 135 (23): 2033-2040. Disponible en: https://watermark.silverchair.com/bloodbld2020006000c.pd f?token=AQECAHi208BE49Ooan9kkhW_Ercy7Dm3ZL_9 Cf3qfKAc485ysgAAA5YwggOSBgkqhkiG9w0BBwagggOD MIIDfwIBADCCA3gGCSqGSIb3DQEHATAeBglghkgBZQM EAS4wEQQMIdh6BiJ17AdXg5OnAgEQgIIDSQH3Uekf0-OKam1oviDuxBcdJ0P7WmvBOH

22. Zou $\mathrm{H}$, Xiong WF. Advances in the relationship between coronavirus infection and coagulation function. Chin Med $\mathrm{J}$ (Engl) [Internet]. 2020; 133 (12): 1489-1490. Disponible en: https://journals.Iww.com/cmj/Citation/9000/Advances_in_ the_relationship_between_coronavirus.99322.aspx

23. Newall F, Branchford B, Male C. Anticoagulant prophylaxis and therapy in children: current challenges and emerging issues. J Thromb Haemost. 2018; 16 (2): 196-208.

24. Whittle JS, Pavlov I, Sacchetti AD, Atwood C, Rosenberg MS. Respiratory support for adult patients with COVID-19. J Am Coll Emerg Physicians Open [Internet]. 2020; 10.1002/ emp2.12071. Disponible en: https://onlinelibrary.wiley.com/ doi/pdf/10.1002/emp2.12071

25. Pastor Vivero MD, Pérez Tarazona S, Rodríguez Cimadevilla JL. Fracaso respiratorio agudo y crónico. Oxigenoterapia. Protoc Diagn Ter Pediatr. 2017; 1: 369-399.

26. Bugarín González R, Martínez Rodríguez JB. La oxigenoterapia en situaciones graves [Internet]. Medicina Integral. 2000; 36 (5): 159-165. Disponible en: https:// www.academia.edu/36414966/La_oxigenoterapia_en_ situaciones graves

27. World Health Organization. Oxygen therapy for children. Geneva: World Health Organization; 2016. pp. 1-57. Disponible en: https://apps.who.int/iris/bitstream/ handle/10665/204584/9789241549554_eng.pdf;jsessionid= 3771BCCE16FF08061123B70DF03F9F4F? sequence $=1$

28. Luna Paredes MC, Asensio De La Cruz O, Cortell Aznar I, Martínez Carrasco MC, Barrio Gómez De Agüero MI, Pérez Ruiz E et al. Oxygen therapy in acute and chronic conditions: Indications, oxygen systems, assessment and follow-up. An Pediatr. 2009; 71 (2): 161-174.

29. Lyons $\mathrm{C}$, Callaghan $\mathrm{M}$. The use of high-flow nasal oxygen in COVID-19. Anaesthesia [Internet]. 2020; 75 (7): 843847. Available from: https://onlinelibrary.wiley.com/doi/ pdf/10.1111/anae.15073

30. Jie L. Expert consensus on preventing nosocomial transmission during respiratory care for critically ill patients infected by 2019 novel coronavirus pneumonia. Chinese $\mathrm{J}$ Tuberc Respir Dis. 2020; 17: E020.

31. Olveira C, Muñoz A, Domenech A. Terapia nebulizada. Año SEPAR. Arch Bronconeumol. 2014; 50 (12): 535-545.

32. Canesí Gómez C, Peñuelas Rodríguez O. Recomendaciones de consenso respecto al soporte respiratorio no invasivo en el paciente adulto con insuficiencia respiratoria aguda secundaria a infección por SARS-CoV-2. Rev Esp Anestesiol Reanim [Internet]. 2020; 67 (5): 261-270. Disponible en: https://www.elsevier.es/esrevista-revista-espanola-anestesiologia-reanimacion-344pdf-S0034935620300785

\section{Correspondencia:}

Dra. Laura Elena Carreto Binaghi

E-mail: lecarreto@iner.gob.mx 